\title{
Avaliação do controle da asma em universitários no sul do Brasil
}

\author{
Evaluation of asthma control in university students in southern Brazil \\ Fernanda Barros Prieto (D), Ana Maria Baptista Menezes (D), Paula Duarte de Oliveira
}

\section{RESUMO}

Objetivo: Mensurar o nível de controle da asma em estudantes com relato de chiado no peito no último ano através do Asthma Control Test (ACT). Método: Estudo transversal descritivo realizado com ingressantes na Universidade Federal de Pelotas (UFPel) em 2017, incluindo 1.865 universitários com 18 anos ou mais. Resultados: Cerca de $19 \%$ dos universitários apresentaram chiado no peito nos 12 meses que antecederam a entrevista. Destes, $14 \%$ apresentaram asma não controlada ( $A C T \leq 18$ pontos). A maior prevalência de asma não controlada foi observada no sexo feminino, em indivíduos com 24 anos ou mais, com a cor da pele parda, em um quarto dos universitários pertencentes às classes $D$ e $E$, e naqueles que não fumam e não consomem bebida alcoólica. Conclusão: Apesar de a população estudada ser composta por universitários, observou-se uma prevalência expressiva de asma não controlada.

Palavras-chave: Asma; Chiado; Adulto Jovem; Saúde do Estudante; Estudos Epidemiológicos.

\begin{abstract}
Objective: To measure the level of asthma control in students who have reported wheezing in the last year through the Asthma Control Test (ACT). Method: A cross-sectional study was carried out with students for the University Federal of Pelotas (UFPel) in 2017, including 1865 university students aged 18 years or over. Results: About $19 \%$ of the interviewees reported wheezing in the last year. Of these, $14 \%$ had uncontrolled asthma (ACT $\leq 18$ points). The highest prevalence of uncontrolled asthma was observed among females, in individuals aged 24 years or older, with brown skin color, in one quarter of university students in class D and E, and who did not smoke and did not consume alcohol. Conclusion: Although the population studied was composed by university students, a considerable percentage of them had uncontrolled asthma.
\end{abstract}

Keywords: Asthma; Wheezing; Young Adult, Student Health; Epidemiologic Studies.

\footnotetext{
Mestre, Programa de Pós-graduação em Epidemiologia, Universidade Federal de Pelotas (RS), Brasil.

PhD, Prof. titular do Programa de Pós-graduação em Epidemiologia, Universidade Federal de Pelotas (RS), Brasil.

PhD, Pós-doutoranda do Programa de Pós-Graduação em Epidemiologia, Universidade Federal de Pelotas (RS), Brasil.

$\triangle$ Fernanda Barros Prieto. Rua Marechal Deodoro, 1160 - 30 piso - Centro. CEP: 96020-220 - Caixa Postal 464. Pelotas (RS), Brasil.

fernandabarros.fisio@gmail.com | Recebido em: 16/02/2020 | Aprovado em: 23/09/2020
} 


\section{INTRODUÇÃO}

A asma é uma doença heterogênea, geralmente caracterizada por inflamação crônica das vias aéreas. É definida por um conjunto de sintomas respiratórios como chiado, tosse e falta de ar, que variam em intensidade e ao longo do tempo, juntamente com uma limitação variável do fluxo expiratório ${ }^{1}$.

A inexistência de um marcador biológico exclusivo da asma e a variabilidade da expressão clínica tornam difícil a avaliação da doença em estudos epidemiológicos ${ }^{2}$. Em razão disso, questionários padronizados e validados como International Study of Asthma and Allergies in Childhood - ISAAC e European Community Respiratory Health Survey ECRHS têm sido amplamente utilizados para medir a prevalência de asma através de sintomas respiratórios em grandes amostras populacionais ${ }^{3,4}$.

O chiado no peito (sibilos) é considerado um sintoma indicativo de asma (proxy), principalmente, em populações jovens. Estudos epidemiológicos internacionais têm avaliado asma através da pergunta sobre presença de sibilos nos últimos 12 meses $^{5}$.

Segundo a Pesquisa Mundial em Saúde (World Health Survey (WHS), 2002-2003), a prevalência global de chiado no peito nos últimos 12 meses foi de $8,6 \%$, considerando a faixa etária de adultos de 18 a 45 anos $^{6}$. O Brasil, um dos vários participantes da WHS, destaca-se entre os cincos países com as mais altas prevalências de chiado no peito $(23 \%)$. No entanto, somente $12 \%$ dos sintomáticos possuíam diagnóstico prévio de asma.

Ainda que existam medicações efetivas para o tratamento da asma desde os anos 80, o controle completo dos sintomas não é obtido pela maioria dos pacientes, sendo esse um dos grandes motivos pelos quais as agudizações persistem como uma das principais causas de atendimentos em serviços de emergência e de hospitalizações ${ }^{7,8}$.

Em adultos, há poucos estudos de amostras representativas da população que tenham avaliado o nível de controle da asma. Isso deve-se a fatores, como: dificuldade na definição de asma mundialmente aceita para estudos epidemiológi$\cos ^{9}$, relato baseado na autopercepção dos sintomas da doença ${ }^{10}$ e o fato de que a asma é mais prevalente na infância e começo da adolescência, sendo que, posteriormente, os sintomas tendem a diminuir, embora saiba-se que a asma pode retornar na idade adulta ${ }^{11}$. Sendo assim, necessita-se que este paradigma seja modificado para que se tenha conhecimento da real dimensão do controle da asma em adultos, e se desenvolvam estratégias de manejo da doença para obtenção do controle dos sintomas pela maioria da população.

Diante desse cenário, o presente estudo teve como objetivo avaliar o nível de controle da asma investigado através do sintoma de chiado nos últimos 12 meses e descrever suas características, em uma população universitária no sul do Brasil.

\section{MATERIAL E MÉTODOS}

Estudo transversal, descritivo, realizado entre novembro de 2017 a julho de 2018 com estudantes da Universidade Federal de Pelotas (UFPel). Os alunos elegíveis deveriam ter 18 anos ou mais, serem ingressantes no primeiro semestre de 2017 e estarem matriculados em cursos de graduação de $2017 / 2$. O presente estudo fez parte de uma pesquisa sobre aspectos gerais em saúde dos universitários da UFPel, realizado na forma de "Consórcio de Pesquisa"12, uma estratégia onde vários mestrandos trabalham em conjunto para que seus dados sejam coletados através de um único instrumento, otimizando o processo e diminuindo custos.

Após a universidade disponibilizar os nomes, os números de matrículas e as disciplinas que os estudantes estavam cursando, foram realizados contatos com os coordenadores e professores dos 80 cursos presenciais para solicitar um período da aula em que pudesse ser aplicado o questionário.

Posteriormente, os mestrandos formaram equipes e dirigiram-se às salas de aula para a apresentação e aplicação da pesquisa; os alunos inelegíveis (menores de 18 anos ou com ingresso em outro semestre), bem como as recusas, eram detectados e liberados da aula. A coleta de dados foi realizada em tablets, através de questionários autoaplicáveis programados no software Research Eletronic Data Capture (RedCap $)^{13}$. Nesta pesquisa optou-se pelo anonimato, não sendo possível efetuar controle de qualidade. $\mathrm{O}$ questionário foi composto por 
perguntas referentes a dados demográficos, socioeconômicos, comportamentais e por questões específicas relacionadas a cada dissertação.

O Asthma Control Test (ACT), teste específico para avaliar o nível de controle da asma, foi aplicado naqueles que relataram "chiado no peito nos

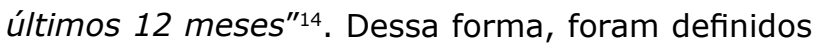
pacientes com sintomas de asma somente aqueles que responderam positivamente à pergunta de chiado no peito no último ano.

Neste estudo, o escore do ACT foi utilizado de duas formas: a) pontuação total do questionário variando de cinco a 25 pontos, para avaliação do desfecho de forma contínua; b) desfecho dicotômico, com ponto de corte de 18 pontos ou menos para caracterizar a asma não controlada, de acordo com a maior sensibilidade e especificidade demonstrados em estudo de validação prévio ${ }^{15}$.

Para as variáveis de chiado no peito e de asma não controlada foram estudadas as prevalências das seguintes variáveis: sexo, idade $(<20$, 20 a 23 e 24 anos ou mais), cor da pele (branca, preta, parda e outras - que engloba amarela e indígena), índice de bens - conforme os critérios da ABEP (Associação Brasileira de Empresas de Pesquisa $)^{16}$, escolaridade materna em níveis completos de estudo (analfabeta, ensino fundamental, ensino médio e ensino superior), tabagismo (nunca fumou, ex-fumante - não fuma há mais de um mês, fumante - fuma pelo menos um cigarro por dia há mais de um mês), número de cigarros fumados por dia (um a cinco, seis a dez e mais de dez) e uso de álcool, segundo o Alcohol Use Disorder Identification Test (AUDIT) (não consome, consumo de baixo risco, uso de risco, uso nocivo e provável dependência) ${ }^{17}$.

A descrição dos achados foi realizada através de frequências absolutas e relativas com seus respectivos intervalos de confiança de $95 \%$. Foi utilizado o teste do qui-quadrado de tendência linear para as variáveis categóricas ordinais que não apresentaram desvio de linearidade e para as demais variáveis foi utilizado o teste do qui-quadrado de heterogeneidade. A variável contínua do ACT apresentou distribuição assimétrica, portanto as diferenças entre as médias de pontuação do ACT conforme as categorias das variáveis independentes foram verificadas pelo teste não paramétrico de Kruskal-Wallis. A análise dos dados foi realizada com o pacote estatístico Stata/IC 15.1 (Stata Corp., College Station, TX, EUA).

Os participantes assinaram um termo de consentimento livre e esclarecido prévio ao início do preenchimento do questionário. O projeto geral foi aprovado pelo Comitê de Ética em Pesquisa da Faculdade de Medicina da UFPel em outubro de 2017, sob o número 79250317.0.0000.5317.

\section{RESULTADOS}

Dos 2.706 indivíduos elegíveis, 1.865 compuseram a amostra, sendo $45 \%$ dos participantes do sexo masculino. Houveram $31 \%$ de perdas e recusas, em que a maioria das perdas são justificadas pela desistência dos estudantes ao longo do ano letivo.

Mais de $70 \%$ da amostra tinham até 23 anos e cor da pele branca; $80 \%$ dos estudantes pertenciam às classes sociais $B$ e $C$, conforme os critérios da ABEP. Em torno de $11 \%$ eram fumantes, sendo que aproximadamente metade destes costumavam consumir 6 ou mais cigarros por dia, e $5 \%$ dos universitários faziam uso nocivo de álcool ou tinham provável dependência (Tabela 1).

Da amostra total, 360 (19,5\%; IC 95\% $17,7-21,3)$ relataram chiado no peito no último ano. Entre os 351 respondentes do ACT ( 9 indivíduos não responderam todo o questionário), $52(14,5 \%$; IC $95 \% 11,2-18,6)$ apresentaram asma não controlada ( $A C T \leq 18$ pontos) e a pontuação média total dos testes foi de 22,2 (DP 3,6) e 21,7 (DP 3,1) pontos, em homens e mulheres respectivamente (Tabela 1 ).

Menos da metade da amostra (39\% das mulheres e $45 \%$ dos homens) relatou que sua doença esteve completamente controlada durante as últimas quatro semanas (Figura 1). As mulheres em relação aos homens relataram maior prejuízo em suas atividades de vida diária, (6\% responderam "na maioria das vezes/todo o tempo", contra $2 \%$, respectivamente). As universitárias também relataram maior ocorrência de falta de ar pelo menos uma vez ao dia, $8 \%$ (sendo $1 \%$ nos homens). 


\section{Tabela 1}

Descrição da amostra de universitários total e por sexo, segundo características sociodemográficas, comportamentais e de saúde.

\begin{tabular}{|c|c|c|c|c|c|c|}
\hline \multirow{3}{*}{ Variáveis } & \multicolumn{3}{|c|}{ Amostra total } & \multicolumn{3}{|c|}{ Sexo } \\
\hline & \multicolumn{3}{|c|}{ Masculino } & \multicolumn{3}{|c|}{ Feminino } \\
\hline & $\mathrm{n}$ & $\%$ & $\mathrm{n}$ & $\%$ & $\mathrm{n}$ & $\%$ \\
\hline \multicolumn{7}{|l|}{ Idade d } \\
\hline$<20$ & 768 & 41,5 & 327 & 39,2 & 440 & 43,3 \\
\hline $20-23$ & 705 & 38,0 & 329 & 39,5 & 375 & 37,0 \\
\hline$\geq 24$ & 379 & 20,5 & 178 & 21,3 & 200 & 19,7 \\
\hline \multicolumn{7}{|l|}{ Cor da pele d } \\
\hline Branca & 1.343 & 72,1 & 605 & 72,0 & 735 & 72,1 \\
\hline Preta & 242 & 13,0 & 104 & 12,4 & 138 & 13,5 \\
\hline Parda & 247 & 13,3 & 113 & 13,5 & 134 & 13,1 \\
\hline Outras & 31 & 1,7 & 18 & 2,1 & 13 & 1,3 \\
\hline \multicolumn{7}{|l|}{ Índice de bens ad } \\
\hline A & 266 & 14,9 & 136 & 17,1 & 130 & 13,2 \\
\hline B & 787 & 44,2 & 357 & 45,0 & 429 & 43,6 \\
\hline C & 649 & 36,5 & 266 & 33,5 & 382 & 38,8 \\
\hline D-E & 78 & 4,4 & 35 & 4,4 & 43 & 4,4 \\
\hline \multicolumn{7}{|l|}{ Escolaridade materna d } \\
\hline Analfabeta /Ens. Fund. incompleto & 415 & 22,4 & 176 & 21,5 & 239 & 23,5 \\
\hline Ens. Fundamental & 222 & 12,0 & 91 & 10,9 & 130 & 12,8 \\
\hline Ens. Médio & 595 & 32,1 & 259 & 31,1 & 334 & 32,8 \\
\hline Ens. Superior & 622 & 33,5 & 306 & 36,8 & 316 & 31,0 \\
\hline \multicolumn{7}{|l|}{ Tabagismo d } \\
\hline Nunca fumou & 1.369 & 73,5 & 593 & 70,5 & 775 & 75,9 \\
\hline Ex-fumante & 290 & 15,6 & 134 & 15,9 & 156 & 15,3 \\
\hline Fumante & 204 & 10,9 & 114 & 13,6 & 90 & 8,8 \\
\hline \multicolumn{7}{|l|}{ Número de cigarros/dia d } \\
\hline $1-5$ & 109 & 54,2 & 58 & 52,7 & 51 & 56,0 \\
\hline $6-10$ & 51 & 25,4 & 30 & 27,3 & 21 & 23,1 \\
\hline$>10$ & 41 & 20,4 & 22 & 20,0 & 19 & 20,9 \\
\hline \multicolumn{7}{|l|}{ Uso de álcool bd } \\
\hline Não consome & 150 & 8,1 & 51 & 6,1 & 99 & 9,7 \\
\hline Uso de baixo risco & 1.140 & 61,4 & 505 & 60,1 & 634 & 62,3 \\
\hline Uso de risco & 476 & 25,6 & 231 & 27,5 & 245 & 24,1 \\
\hline Uso nocivo/Provável dependência & 92 & 4,9 & 53 & 6,3 & 39 & 3,8 \\
\hline Diagnóstico de asma na vidad & 444 & 23,9 & 222 & 26,6 & 222 & 21,9 \\
\hline Chiado no peito no último ano ${ }^{d}$ & 360 & 19,5 & 146 & 17,5 & 214 & 21,1 \\
\hline $\mathrm{ACT} \leq 18$ pontos $^{\mathrm{cd}}$ & 51 & 14,5 & 19 & 13,3 & 32 & 15,4 \\
\hline Média do ACT (DP) cd & 21,9 & 3,5 & 22,2 & 3,6 & 21,7 & 3,1 \\
\hline Total & 1.865 & 100 & 841 & 45,2 & 1.021 & 54,8 \\
\hline
\end{tabular}

ACT - Asthma Control Test a Classificação conforme ABEP (Associação Brasileira de Empresas de Pesquisa) ${ }^{\mathrm{b}}$ Classificação conforme AUDIT (Alcohol Use Disorder Identification Test) ${ }^{\mathrm{c}}$ Responderam ao ACT apenas aqueles que referiram chiado no peito no último ano, sendo nove perdas por não completar o questionário d Maior perda de informação para a variável índice de bens com $\mathrm{n}=85$ para ignorados. 


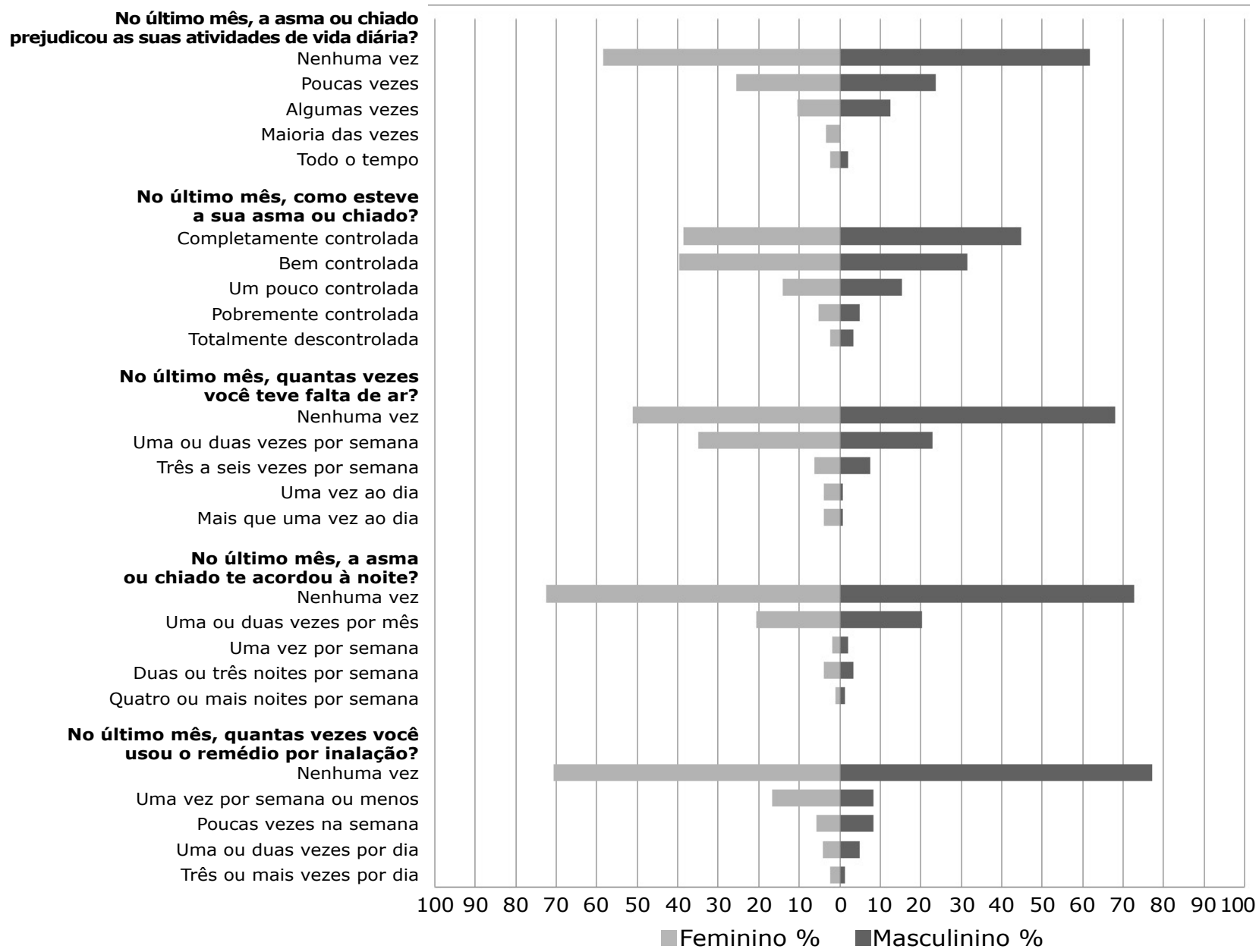

Figura 1. Distribuição das respostas ao ACT estratificada por sexo.

Do total dos respondentes, 6,5\% utilizaram o medicamento para alívio do chiado uma ou mais vezes ao dia (Tabela Suplementar); entre os estudantes com asma não controlada, cerca de $22 \%$ não utilizaram o medicamento de resgate no último mês (dado não apresentado em tabelas). O percentual de respostas para cada alternativa das questões do ACT encontra-se na Tabela Suplementar.

A presença de chiado no peito no último ano, prevalência de ACT $\leq 18$ pontos e média de pontuação no ACT conforme características da amostra encontram-se na Tabela 2.

Tabela Suplementar

Resultados das questões do Asthma Control Test (ACT) por categoria de resposta $(n=351)$

Questões

Respostas \%

1. No último mês, a asma ou bronquite ou chiado prejudicou as suas atividades no local de estudo, trabalho ou em casa? d

(5) Nenhuma vez

(4) Poucas vezes

(3) Algumas vezes

11,3

(2) Maioria das vezes 
Tabela Suplementar (Continuação)

Questões

Respostas \%

(1) Todo o tempo 2,3

2. No último mês, como esteve a sua asma, bronquite ou chiado? ${ }^{\mathrm{d}}$

(5) Completamente controlada 41,1

(4) Bem controlada 36,3

(3) Um pouco controlada 14,6

(2) Pobremente controlada 5,1

(1) Totalmente descontrolada 2,9

3. No último mês, quantas vezes você teve falta de ar? d

(5) Nenhuma vez 58,0

(4) Uma ou duas vezes por semana 30,0

(3) Três a seis vezes por semana $\quad 6,8$

(2) Uma vez ao dia $\quad 2,6$

(1) Mais que uma vez ao dia 2,6

4. No último mês, a sua asma ou bronquite ou chiado te acordou à noite ou mais cedo que de costume? ${ }^{d}$

(5) Nenhuma vez $\quad 72,7$

$\begin{array}{ll}\text { (4) Uma ou duas vezes } & 20,5\end{array}$

(3) Uma vez por semana $\quad 2,0$

(2) Duas ou três noites por semana $\quad 3,7$

(1) Quatro ou mais noites por semana 1,1

5. No último mês, quantas vezes você usou remédio por inalação (ou bombinha) para alívio da asma ou bronquite ou chiado? d

(5) Nenhuma vez

(4) Uma vez por semana ou menos

(3) Poucas vezes na semana

(2) Uma ou duas vezes por dia

4,6

(1) Três ou mais vezes por dia

1,9

${ }^{\mathrm{d}}$ Questões com dados faltantes - máximo de 10 observações para a questão $\mathrm{n}^{\circ} 2$.

\section{Tabela 2}

Descrição da presença de chiado no peito no último ano, prevalência do Asthma Control Test (ACT) $\leq 18$ pontos e média de pontuação no ACT, conforme características da amostra de universitários.

\begin{tabular}{|c|c|c|c|c|c|c|}
\hline \multirow[t]{2}{*}{ Variáveis } & \multicolumn{2}{|c|}{$\begin{array}{l}\text { Prevalência de chiado no } \\
\text { peito no último ano }\end{array}$} & \multicolumn{2}{|c|}{$\begin{array}{l}\text { Asma não controlada } \\
\text { (ACT } \leq 18 \text { pontos) }\end{array}$} & \multicolumn{2}{|l|}{ Média ACT + } \\
\hline & $\%$ & IC 95\% & $\%$ & IC 95\% & Média & DP \\
\hline Sexo $^{c}$ & $p=0,052$ & & $p=0,584$ & & $p=0,113$ & \\
\hline Masculino & 17,5 & $15,1-20,2$ & 13,3 & $8,6-19,9$ & 22,2 & 3,6 \\
\hline Feminino & 21,1 & $18,7-23,7$ & 15,4 & $11,1-20,9$ & 21,7 & 3,1 \\
\hline Idade $^{c}$ & $p=0,118$ & & $\mathrm{p}=0,039 *$ & & $p=0,314$ & \\
\hline$<20$ & 21,5 & $18,7-24,6$ & 11,1 & $7,1-17,0$ & 22,2 & 3,1 \\
\hline $20-23$ & 17,2 & $14,6-20,2$ & 15,3 & $9,8-23,0$ & 21,9 & 3,3 \\
\hline$\geq 24$ & 19,6 & $15,9-24,0$ & 21,7 & $13,5-33,0$ & 21,1 & 4,5 \\
\hline Cor da pele ${ }^{c}$ & $p=0,171$ & & $p=0,230$ & & $p=0,341$ & \\
\hline Branca & 18,2 & $16,2-20,4$ & 11,9 & $8,3-16,7$ & 22,1 & 3,2 \\
\hline Preta & 23,1 & $18,3-28,9$ & 18,5 & $10,2-31,2$ & 21,7 & 3,4 \\
\hline Parda & 22,7 & $17,9-28,3$ & 21,4 & $12,6-34,1$ & 21,1 & 4,0 \\
\hline Outra & 19,4 & $9,0-37,0$ & 20,0 & $2,7-69,3$ & 21,6 & 4,5 \\
\hline
\end{tabular}


Tabela 2 (Continuação)

\begin{tabular}{|c|c|c|c|c|c|c|}
\hline \multirow{2}{*}{$\begin{array}{l}\text { Variáveis } \\
\text { Índice de bens ac }\end{array}$} & \multicolumn{2}{|c|}{$\begin{array}{l}\text { Prevalência de chiado no } \\
\text { peito no último ano }\end{array}$} & \multicolumn{2}{|c|}{$\begin{array}{l}\text { Asma não controlada } \\
\text { (ACT } \leq 18 \text { pontos) }\end{array}$} & \multicolumn{2}{|l|}{ Média ACT + } \\
\hline & $p=0,458$ & & $p=0,374$ & & $p=0,279$ & \\
\hline$A$ & 18,6 & $14,3-23,7$ & 8,2 & $3,1-20,0$ & 22,3 & 3,6 \\
\hline $\mathrm{B}$ & 17,9 & $15,4-20,8$ & 13,9 & $9,1-21,0$ & 22,0 & 3,3 \\
\hline $\mathrm{C}$ & 21,2 & $18,3-24,6$ & 15,2 & $10,0-22,3$ & 21,8 & 3,4 \\
\hline$D-E$ & 20,5 & $13,0-31,0$ & 25,0 & $9,7-50,9$ & 20,6 & 4,0 \\
\hline Escolaridade materna ${ }^{c}$ & $p=0,159$ & & $p=0,070$ & & $p=0,478$ & \\
\hline Analfabeta/ Ens. Fund. incompleto & 18,5 & $15,0-22,5$ & 12,7 & $6,7-22,6$ & 22,1 & 3,6 \\
\hline Ens. Fundamental & 14,5 & $10,5-19,9$ & 32,3 & $18,3-50,3$ & 21,0 & 3,7 \\
\hline Ens. Médio & 21,2 & $18,1-24,7$ & 15,4 & $10,0-23,0$ & 21,8 & 3,5 \\
\hline Ens. Superior & 20,6 & $17,5-24,0$ & 10,3 & $6,1-17,0$ & 22,2 & 3,2 \\
\hline Tabagismo $^{c}$ & $p<0,001$ & & $p=0,453$ & & $p=0,799$ & \\
\hline Nunca fumou & 17,7 & $15,7-19,8$ & 15,8 & $11,7-21,1$ & 21,9 & 3,4 \\
\hline Ex-fumante & 16,3 & $12,5-21,0$ & 8,7 & $3,3-21,0$ & 22,3 & 3,5 \\
\hline Fumante & 36,1 & $30,0-43,0$ & 14,1 & $7,7-24,3)$ & 21,8 & 3,5 \\
\hline Número de cigarros/dia c & $p=0,679$ & & $p=0,042 *$ & & $p=0,280$ & \\
\hline $1-5$ & 34,6 & $26,1-44,1$ & 8,3 & $2,6-23,2$ & 22,2 & 2,8 \\
\hline $6-10$ & 33,3 & $21,8-47,3$ & 11,8 & $2,9-37,5$ & 22,5 & 3,1 \\
\hline$>10$ & 41,5 & $27,5-57,0$ & 31,3 & $13,4-57,1$ & 19,9 & 4,9 \\
\hline Uso de álcool bc & $\mathrm{p}<0,001 *$ & & $p=0,458$ & & $p=0,350$ & \\
\hline Não consome & 17,6 & $12,2-24,6$ & 23,1 & $10,7-42,8$ & 20,6 & 5,0 \\
\hline Baixo risco & 17,2 & $15,1-19,6$ & 15,4 & $10,9-21,3$ & 21,8 & 3,4 \\
\hline Uso de risco & 22,8 & $19,3-26,8$ & 11,3 & $6,5-18,9$ & 22,4 & 2,9 \\
\hline Uso nocivo/Provável dependência & 33,7 & $24,8-43,9$ & 12,9 & $4,9-30,0$ & 22,0 & 3,3 \\
\hline Total & 19,5 & $17,7-21,3$ & 14,5 & $11,2-18,6$ & 21,9 & 3,4 \\
\hline
\end{tabular}

${ }^{a}$ Conforme classificação ABEP (Associação Brasileira de Empresas de Pesquisa) ${ }^{\text {b }}$ Conforme AUDIT (Alcohol Use Disorder Identification Test) ${ }^{\mathrm{c}}$ Maior perda de informação para a variável índice de bens com $\mathrm{n}=85$ para ignorados. ${ }^{*}$ Teste do qui-quadrado de tendência linear, restante Teste do qui-quadrado de heterogeneidade. ${ }^{+}$Diferenças entre médias do ACT por categorias verificadas pelo teste não paramétrico de Kruskal-Wallis; $\mathrm{N}$ respondentes sobre chiado no peito no último ano=360; $\mathrm{N}$ que apresentou chiado no peito no último ano e respondeu ao $\mathrm{ACT}=351$.

Quanto ao chiado no peito, a maior prevalência foi entre o sexo feminino, em menores de 20 anos, em indivíduos de cor da pele preta, pertencentes à classe $C$ e filhos de mães com ensino médio completo. Tabagistas apresentaram maior prevalência de chiado no peito, 36,1\% (IC 95\% 29,8 - 43,0; $p<0,001$ ) e universitários com uso nocivo de álcool $(33,7 \%$; IC 95\% 24,7 - 43,9; p < 0,001) (Tabela 2).

A maior prevalência de asma não controlada foi observada no sexo feminino, com a cor da pele parda, e em um quarto dos universitários pertencentes à classe $\mathrm{D}$ e E. Filhos de mães com ensino superior completo apresentaram menor prevalência de asma não controlada. Universitários com 24 anos ou mais (21,7\%; IC 95\% 13,5-33,0; $\mathrm{p}=0,039)$ e tabagistas que consomem mais de 10 cigarros por dia (31,3\%; IC $95 \% 13,4-57,1$; $p=0,042)$ apresentaram significativamente maior prevalência de asma não controlada. E naqueles que não fumam e não consomem álcool foi observada maior prevalência de asma não controlada. Não houve significância estatística para as diferenças entre as médias de pontuação no ACT conforme as características dos universitários (Tabela 2). 


\section{DISCUSSÃO}

No presente estudo foi demonstrado que a população de universitários, predominantemente composta de adultos jovens, apresentou prevalência expressiva de asma não controlada e presença de chiado no peito no último ano. Aqueles que fumam e fazem uso nocivo de álcool apresentaram significativamente maior prevalência de chiado; aqueles que não fumam e não consomem álcool demonstraram maior proporção de asma não controlada.

A prevalência de chiado no peito no último ano foi $19,5 \%$, valor superior ao observado na literatura para este mesmo desfecho considerando o autorrelato de jovens adultos. Recentes estudos epidemiológicos realizados em países em desenvolvimento como o Brasil encontraram prevalências de: $13,9 \%$ (Argentina) $^{18}, 13,1 \%$ (Irã) ${ }^{19}$ e $12,1 \%$

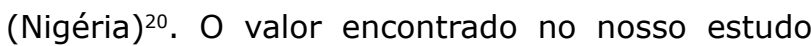
aproxima-se com os achados da WHS $(22,6 \%)$, em que o Brasil se destaca com uma das mais altas prevalências entre os 70 países avaliados ${ }^{6}$.

A asma não controlada foi observada em $14,5 \%$ dos universitários com relato de chiado no último ano, valor inferior ao encontrado em alguns estudos que também utilizaram o ACT para avaliar o nível de controle da asma. Um estudo polonês com população universitária encontrou $19,0 \%$ de asma não controlada ${ }^{21}$, enquanto um inquérito realizado na Nova Zelândia e na Austrália relataram 21,8\% e $22,9 \%$, respectivamente, para o mesmo desfecho ${ }^{22}$.

No entanto, a comparação entre os estudos que adotaram o ACT como instrumento torna-se difícil, devido ao fato de que grande parte das pesquisas foram desenvolvidas em ambulatórios e/ou hospitais de países de alta renda e com diferenças metodológicas na definição de asma; em grande parte destes estudos, o nível de controle da asma foi classificado de forma distinta, com outros pontos de corte ${ }^{23-25}$. Através da revisão de literatura verificou-se uma variação de prevalência de asma não controlada na população adulta de $14,5 \%$ a $68,1 \%{ }^{26,27}$. Como citado anteriormente na metodologia, optamos pelo ponto de corte conforme o estudo de validação do ACT para a língua portugue$\mathrm{sa}^{15}$, e o cálculo do tamanho da amostra considerou apenas duas categorias de controle da asma (controlada ou não controlada).
Em nossos achados, mulheres de classes sociais mais baixas relataram mais chiado no peito e somaram menos pontos no ACT. Estudos anteriores mostraram que mulheres referem sintomas respiratórios frequentemente, visitas regulares a serviços de saúde e apresentam menor grau de controle da asma ${ }^{29}$. Apesar de homens e mulheres asmáticos compartilharem características comuns da doença, a história natural da asma difere entre os $\operatorname{sexos}^{30}$. Alguns autores sugerem que o fator hormonal parece ser um componente fundamental para o entendimento das diferenças observadas entre os sexos ${ }^{29,31}$.

Universitários tabagistas que consomem mais de dez cigarros por dia e que fazem uso nocivo de álcool apresentaram maior prevalência de chiado no peito no último ano. Alguns estudos citam que aqueles que fumam mais, também são os que fazem uso abusivo de álcool e, consequentemente, têm menor cuidado com a saúde ${ }^{32,33}$. Em nossa amostra, 20,3\% dos universitários com chiado no peito faziam consumo simultâneo de tabaco e álcool.

Em direção oposta ao esperado, aqueles estudantes que relataram não fumar e não beber apresentaram maior prevalência de asma não controlada. Porém, deve-se considerar o possível viés de causalidade reversa - indivíduos com asma mais grave, portanto de difícil controle, não fumam e não consomem álcool. Outra hipótese para este achado é de que os universitários com sintomas mais exacerbados subnotificaram o hábito de fumar e de consumir álcool ${ }^{34}$.

Quanto às limitações do nosso estudo, uma delas foi a impossibilidade de mensurar as causas de ausência às aulas. Possivelmente, universitários com sintomas respiratórios exacerbados, e, portanto, com a asma não controlada, sejam aqueles que mais faltem às aulas, ocasionando viés de seleção, subestimando a real prevalência dos desfechos estudados. Ademais, apesar da ampla aplicação da definição de casos de asma a partir do relato de chiado em estudos epidemiológicos, deve-se ter clara a possibilidade de abrangência a alguns indivíduos com diagnósticos alternativos à doença. Adicionalmente, o tamanho da amostra talvez não tenha permitido detectar algumas associações pela falta de poder, assim como deve ser ressaltado o elevado número de perdas, devido às desistências dos universitários ao longo do semestre letivo. 


\section{CONCLUSÃO}

Diferente da maior parte dos estudos encontrados na literatura, o nosso trabalho teve a oportunidade de retratar a prevalência dos desfechos estudados em jovens adultos da população em geral, e não naqueles presentes nos serviços de saúde. Numerosos estudos epidemiológicos são relatados em crianças e adolescentes, entretanto, a população adulta jovem tem sido pouco avaliada no que se refere ao controle da asma, dificultando a realização de comparações e conhecimento sobre esse público. São necessárias futuras pesquisas nessa faixa etária, com outros tipos de delineamentos, para detectar associações e temporalidade.

A utilização de um instrumento como o ACT para avalição do controle da asma deve ser encorajada por ser uma ferramenta fácil de administrar e interpretar, de rápida aplicação e sem custo, sendo possível utilizá-la em qualquer ambiente; além disto já foi validada no nosso país. Em particular, quando a espirometria muitas vezes não está disponível, este instrumento pode orientar quanto a condutas futuras relativas à asma e identificar grupos prioritários para intervenções de saúde pública no que tange à prevenção e o tratamento, objetivando o controle da asma.

\section{Órgão financiador da pesquisa}

O presente trabalho foi realizado com apoio da Coordenação de Aperfeiçoamento de Pessoal de Nível Superior - Brasil (CAPES) - Código de Financiamento 001 e Programa de Excelência Acadêmica (PROEX). AMBM é bolsista de Produtividade em Pesquisa 1A (CAPES).

\section{REFERÊNCIAS}

1. Horak, Fritz et al. Diagnosis and management of asthmaStatement on the 2015 GINA Guidelines. Wiener Klinische Wochenschrift, v. 128, n. 15-16, p. 541-554, 2016.

2. Westerhof GA, Vollema EM, Weersink EJ, Reinartz SM, de Nijs SB, Bel EH. Predictors for the development of progressive severity in new-onset adult asthma. J Allergy Clin Immunol. 2014;134(5):1056-1056.
3. Pizzichini, M. M. M. (2005). Definir asma para estudos epidemiológicos: essa meta pode ser alcançada?. Jornal Brasileiro de Pneumologia, 31(6), vi-viii.

4. Asher, M. I., Keil, U., Anderson, H. R., Beasley, R., Crane, J., Martinez, F., ... \& Stewart, A. W. (1995). International Study of Asthma and Allergies in Childhood (ISAAC): rationale and methods. European respiratory journal, $8(3), 483-491$.

5. Burney, P. G., Luczynska, C., Chinn, S., \& Jarvis, D. (1994). The European community respiratory health survey. European respiratory journal, 7(5), 954-960.

6. Global Asthma Network. The Global Asthma Report 2018. Auckland: New Zealand; 2018.

7. To T, Stanojevic S, Moores G, Gershon AS, Bateman ED, Cruz AA, et al. Global asthma prevalence in adults: findings from the cross-sectional World Health Survey. BMC Public Health. 2012;12(1):204.

8. Diretrizes da Sociedade Brasileira de Pneumologia e Tisiologia para o manejo da asma-2012. J Bras Pneumol. 2012;38 Suppl 1: S1-S46.

9. Gazzotti MR, Nascimento OA, Montealegre F, Fish J, Jardim JR. Nível de controle da asma e seu impacto nas atividades de vida diária em asmáticos no Brasil. J Bras Pneumol. 2013;39(5):532-8.

10. Papi A, Brightling C, Pedersen SE, Reddel HK. Asthma. Lancet. 2018;391(10122):783-800.

11. Ponte EV, Petroni J, Ramos DC, Pimentel L, Freitas DN, Cruz AA. A percepção do controle dos sintomas em pacientes asmáticos. J Bras Pneumol. 2007;33(6):635-40.

12. Westerhof GA, Vollema EM, Weersink EJ, Reinartz SM, de Nijs SB, Bel EH. Predictors for the development of progressive severity in new-onset adult asthma. J Allergy Clin Immunol. 2014;134(5):1056-1056.

13. Barros $A J$, Menezes $A M B$, Santos IS, Assunção $M C F$, Gigante D, Fassa AG, et al. O Mestrado do Programa de Pós-graduação em Epidemiologia da UFPel baseado em consórcio de pesquisa: uma experiência inovadora. Rev Bras Epidemiol. 2008; 11:133-44.

14. Harris PA, Taylor R, Thielke R, Payne J, Gonzalez N, Conde JG. Research electronic data capture (REDCap) - a metadata-driven methodology and workflow process for providing translational research informatics support. J Biomed Inform. 2009;42(2):377-81.

15. Nathan RA, Sorkness CA, Kosinski M, Schatz M, Li JT, Marcus $\mathrm{P}$, et al. Development of the asthma control test: a survey for assessing asthma control. J Allergy Clin Immunol. 2004;113(1):59-65.

16. Roxo JP, Ponte EV, Ramos DC, Pimentel L, D'Oliveira Junior A, Cruz AA. Validação do Teste de Controle da Asma em português para uso no Brasil. J Bras Pneumol. 2010;36(2):159-66.

17. Pesquisa ABEP. Critério de Classificação econômica Brasil; [acesso28nov. 2018]. Disponívelem: http://www.abep.org/ criterio-brasil. 
18. Babor T, Higgins-Biddle J, Saunders J, Monteiro M. AUDIT: teste para identificação de problemas relacionados ao uso de álcool: roteiro para uso em atenção primária. Ribeirão Preto: PAI-PAD, 2003.

19. Arias SJ, Neffen $H$, Bossio JC, Calabrese CA, Videla AJ, Armando GA, et al. Prevalence and Features of Asthma in Young Adults in Urban Areas of Argentina. Arch Bronconeumol. 2018;54(3):134-9.

20. Idani $E$, Raji $H$, Madadizadeh $F$, Cheraghian $B$, Shoshtari $\mathrm{MH}$, Dastoorpoor M. Prevalence of asthma and other allergic conditions in adults in Khuzestan, southwest Iran, 2018. BMC Public Health. 2019;19(1):303.

21. Desalu OO, Sanya EO, Adeoti AO, Aderibigbe SA, Kolo PM. Impact of Operational Definitions on the Predictors and Prevalence of Asthma Estimates: Experience from a University Students' Survey and Implications for Interpretation of Disease Burden. Ethiop J Health Sci. 2018;28(6): 725 .

22. Romantowski J, Gawinowska M, Cyrny P, Jassem $E_{\text {, }}$ Chelminska $M$, Niedoszytko $M$. Asthma prevalence and risk factors analysis in Tricity university students' group. Pneumonol Alergol Pol. 2015;83(5):359-64.

23. Reddel HK, Beckert $L$, Moran $A$, Ingham $T$, Ampon RD, Peters MJ, et al. Is higher population-level use of ICS/ LABA combination associated with better asthma outcomes? Cross-sectional surveys of nationally representative populations in New Zealand and Australia. Respirology. 2017;22(8):1570-8.

24. Corrado A, Renda T, Polese G, Rossi A. Assessment of asthma control: the SERENA study. Respir Med. 2013;107(11):1659-66.

25. Serugendo AN, Kirenga BJ, Hawkes M, Nakiyingi $L$, Worodria W, Okot-Nwang M. Evaluation of asthma control using Global Initiative for Asthma criteria and the Asthma Control Test in Uganda. Int J Tuberc Lung Dis. $2014 ; 18(3): 371-6$.

26. Ciprandi G, Gallo F, Ricciardolo FL. A real-life comparison of the Asthma Control Test and Global Initiative for Asthma asthma control grading. Ann Allergy Asthma Immunol. 2016;117(6):725-7.
27. Dursun $A B$, Kurt OK, Bayiz H, Ozkan E, Cakaloglu A, Karasoy D. Does gender affect asthma control in adult asthmatics? Chron Respir Dis. 2014;11(2):83-7.

28. BinSaeed AA. Asthma control among adults in Saudi Arabia. Study of determinants. Saudi Med $\mathrm{J}$. 2015;36(5):599-604.

29. Zhong N, Lin J, Zheng J, Lai K, Xie C, Tang KJ, et al. Uncontrolled asthma and its risk factors in adult Chinese asthma patients. Ther Adv Respir Dis. 2016;10(6):507-17.

30. Zillmer LR, Gazzotti MR, Nascimento OA, Montealegre F, Fish J, Jardim JR. Diferenças entre os sexos na percepção de asma e sintomas respiratórios em uma amostra populacional em quatro cidades brasileiras. J Bras Pneumol. 2014;40(6):591-8.

31. Forte GC, Hennemann ML, Dalcin PdTR. Controle da asma, função pulmonar, estado nutricional e qualidade de vida relacionada à saúde: diferenças entre homens e mulheres adultos com asma. J Bras Pneumol. 2018;44(4):273-8.

32. Takeda M, Tanabe M, Ito W, Ueki S, Konnno Y, Chihara M, et al. Gender difference in allergic airway remodelling and immunoglobulin production in mouse model of asthma. Respirology. 2013;18(5):797-806.

33. Costa JSDd, Silveira MF, Gazalle FK, Oliveira SS, Hallal PC, Menezes AMB, et al. Consumo abusivo de álcool e fatores associados: estudo de base populacional. Rev Saúde Pública. 2004; 38:284-91.

34. Elicker E, Palazzo LdS, Aerts DRGdC, Alves GG, Câmara S. Uso de álcool, tabaco e outras drogas por adolescentes escolares de Porto Velho-RO, Brasil. Epidem e Serv de Saúde. 2015; 24:399-410.

35. Stelmach $R$, Fernandes FLA, Carvalho-Pinto RM, Athanazio RA, Rached SZ, Prado GF, et al. Comparação entre medidas objetivas do tabagismo e tabagismo autodeclarado em pacientes com asma ou DPOC: será que nossos pacientes dizem a verdade? J Bras Pneumol. $2015 ; 41(2): 124-32.30$. 